Journal of Engineering and Applied Sciences 14 (1): 8-12, 2019

ISSN: 1816-949X

(C) Medwell Journals, 2019

\title{
Sedimentological Method for Nano-Polycomposite Coating
}

\author{
${ }^{1}$ M.N. Mukhtarova, ${ }^{1}$ A.K. Nurseytova, ${ }^{1}$ S.D. Ermaganbetova, ${ }^{1}$ M.T. Beketayeva, \\ ${ }^{1}$ E.M. Zulbuharova, ${ }^{1}$ A.A. Kuikabayeva, ${ }^{1}$ A.K. Danlybayeva, ${ }^{2}$ M.T. Kyzgaryna, \\ ${ }^{2}$ G.K. Nauryzbayeva and ${ }^{2}$ S.N. Sarsenbayeva \\ ${ }^{1}$ Al-Farabi Kazakh National University, Almaty, Kazakhstan Republic \\ ${ }^{2}$ Almaty University of Power Engineering and Telecommunications, \\ Almaty, Kazakhstan Republic
}

\begin{abstract}
The research objective is to develop a method for obtaining a nanosized powder of shungite concentrate for further using as a composite additive to chromium coating. Shungite rocks are unique by composition, structure and features. As of today, shungite is only one natural mineral containing fullerenes and particular organo-mineral complex giving it unusual unique properties which are used in many branches of science, engineering, production including ecology, medicine, agriculture, energy, construction and so on. When using shungite as a dispersion phase to obtain Composite Electrolytic Coatings (CEC) shungite amorphous component will precipitate along with fullerenes providing its specific properties to the coating. Therefore, CEC properties will be conditioned not only by fullerenes present in the CEC composition but also, availability of entire organo-mineral components of shungite. We managed to develop a method of acquiring shungite concentrate consisting of ultra dispersed shungite containing nanoparticles of carbon and all types of oxides included in natural shungite. Based on the review of contemporary state of knowledge about shungite a science based idea of advisability to use shungite as a disperse phase in CEC composition was proposed for the first time.
\end{abstract}

Key words: Composite electrolytic coatings, size of particles, dispersion, diffraction, ultrasonic waves, CEC

\section{INTRODUCTION}

During recent time nanomaterials and nanotechnologies enabling to create materials with unique operational features are of particular interest. Our research objective is to develop a method for obtaining a nanosized powder of shungite concentrate for further using as a composite additive to chromium coating.

Reviews of domestic and international scientific researches show high prospectivity of the following major areas in developing engineering structural materials: manufacturing nano-structural ceramic and precise form composite items, creating nano-structural hard alloys to produce cutting tools with increased wear resisting properties and impact strength, creating nano-structural protective thermo and anticorrosion coatings, creating increased strength and low flammability polymeric composites with fillers made of nanoparticles and nanotubes (Cholodkevich, 1993; Sarsembinov et al., 2006; Yar-Mukhamedov and Yar-Mukhamedova, 2001).

State of the art: Choosing shungite as a material under research is based on the following; Shungite is a black metamorphic rock consisting of organic matter mixed with particles of clay and sand which upon its formation had undergone more or less drastic transformations exposed to high temperatures and pressures (Anonymous, 2014; Volkova and Bogdanova, 1986). Shungite rocks are unique by composition, structure and features. They are unusual by structure natural composite homogeneous distribution of finely dispersed crystalline silicate particles in amorphous silicate matrix. Average size of silicate particles is about $1 \mu$. Composition of various shungite fields is different. For instance, average composition of Zazhoginskoye shungite mine field in Karelia 30\% carbon and $70 \%$ silicates whereas Koksy field in Kazakhstan maximum $15 \%$ carbon and $85 \%$ oxides of various metals. Shungite carbon has amorphous structure stable against graphitation, characterized by high reactivity worth in thermal processes, high sorption and catalytic properties, electric conductivity and chemical stability (Sokolov and Kalinin, 1975).

Whereas shungite carbonic part is answerable for its clearing, sorption properties, then its biological action relates to particularly organo-mineral complex, especially, its water soluble part which includes fullerenes.

Corresponding Author: M.N. Mukhtarova, Al-Farabi Kazakh National University, Almaty, Kazakhstan Republic 


\section{Shungite properties:}

- Density $1800-2000 \mathrm{~g} / \mathrm{cm}^{3}$

- Porosity up to $5 \%$

- Compressive strength 98-117.6 MPa

- Electrical conductivity $1500 \Omega / \mathrm{m}$

- Thermal conductivity coefficient $5 \mathrm{~W} / \mathrm{m} \mathrm{K}$

- Developed internal surface up to $20 \mathrm{~m}^{2} / \mathrm{g}$

- Adsorption activity: by phenol $14 \mathrm{mg} / \mathrm{g}$ by thermolized resins $-20 \mathrm{mg} / \mathrm{g}$ by oil products over $40 \mathrm{mg} / \mathrm{g}$

- $\quad$ Shungite particles have bipolar properties resulting in high adhesion and ability to mix with each and all substances

- $\quad$ Shungite has bactericidal properties

Shungite is about 2 milliard years old but it has not transformed into graphite or adamant and became according to assessments of scientists a new species of carbon existence named fullerene (Cherdyntsev and Kolesnikov, 1965).

Therefore, fullerene is tertiary crystal form (after adamant and graphite) of carbon existence in nature whereas shungite is a composition, mixture of ancient carbonic matter with various non-organic matters-oxides of silica, aluminum, iron and likewise.

As of today, shungite is only one natural mineral containing fullerenes and particular organo-mineral complex giving it unusual unique properties which are used in many branches of science, engineering, production including ecology, medicine, agriculture, energy, construction and so on. Despite of such extensive usage shungite has been never used in resolving such an important challenge as protection of components of machines, mechanisms, aggregates against wear and corrosion by way of applying Composite Electrolytic Coatings (CEC) (Yar-Mukhamedov and Yar-Mukhamedova, 2010; Tseluikin, 2005).

Shungite contains fullerenes-nanosized clusters which may stay in suspended state in suspension for CEC sedimentation for along time, ensuring constancy of suspension density in all volume of non-stirred electrolyte which is a base for obtaining high a quality CEC. Such conditions were achieved only by Richard Grunke in zero-gravity environment that is on the cosmic station board. Exactly, the last event (necessity for creating zero-gravity environment) was the main reason why the method of CEC sedimentation offered by Grunke didn't gain widespread distribution, practice and recognition. In Earth conditions where gravity force is constantly applying, constancy of the suspension density may be achieved only by continuous stirring that is electrolyte movement and this is absolutely intolerable, since, electrolyte movement will not let disperse particles stay on the cathode surface and grow electro-deposition metal.

When using shungite as a dispersion phase to obtain Composite Electrolytic Coatings (CEC) shungite amorphous component will precipitate along with fullerenes providing its specific properties to the coating. Therefore, CEC properties will be conditioned not only by fullerenes present in the CEC composition but also, availability of entire organo-mineral components of shungite.

The shungite density is insignificant, it's less than that of graphite and adamant and makes $1800-2000 \mathrm{~kg} / \mathrm{m}^{3}$. This fact is a positive factor in favor of shungite sedimentation stability in a fine dispersion state in electrolytic chromium plating solution inasmuch as there is no demand for continuous suspension stirring to maintain shungite in a suspended condition. Unlike many disperse particles used for acquiring $\mathrm{CEC}$, shungite disperse particles have bipolar properties. So, at introducing them into electrolyte they ideally mix with the electrolyte and do not create undesirable accumulations and inhomogeneity.

Thus, the shungite unique properties are unquestionable factors capable of resolving many challenges as nano-CEC acquisition technology and also, challenge of their quality cardinal improvement.

Problem statement: We managed to develop a method of acquiring shungite concentrate consisting of ultra dispersed shungite containing nanoparticles of carbon and all types of oxides included in natural shungite. The method essence is that natural shungite containing impurities of predominantly oxides of various elements $\left(\mathrm{Al}_{2} \mathrm{O}_{2}, \mathrm{SiO}{ }_{3} \mathrm{CaO}\right.$ and others $)$ undergoes mechanical grinding followed by sedimentative settling during a certain time period and siphon separation of shungite fine suspension from a large size sediment.

High effectiveness of such shungite separation is ensured by the fact that shungite has (several times less) significantly less density and considerably (by a factor of hundreds) bigger high dispersion ability after size reduction than impurities. Guarantee of the shungite grinding to a high dispersion extent is presence of particles of metal oxides in natural shungite which have very high hardness $\left(\mathrm{Al}_{2} \mathrm{O}_{2}, \mathrm{SiO}_{3}\right.$ with hardness 7-9 Mohs units) whereas the shungite has quite insignificant hardness (about 2 hardness units)

At sedimentation of natural shungite in distilled water impurity particles of it (generally oxides) go through sedimentation faster whereas it itself stays in a suspended state for a longer time. It's important that the longer 
sedimentation time the less coarse particles left in the suspension. Therefore, sedimentation time is a criterion using which particles smaller than a certain size may be selected. However, it should be noted: beginning from a certain size particles quit sedimentation depositing. Those are Brownian particles. Therefore, Brownian particles are the limit under which one can surely talk about maximal sizes of particles selected using sedimentological method.

Shungite deposit recovered as a result of sedimentological settling undergoes multiple treatments in distilled water and sedimentological separation from the settling as described above. This operation isrepeated till a transparent suspension is obtained practically free of shungite. Using this method almost entire shungite and finely-dispersed oxides containing in natural shungite may be isolated in the suspension form.

The recovered suspension undergoes evaporation and the powder concurrently obtained undergoes annealing at temperature $473-523 \mathrm{~K}$. Amount of recovered concentrate as a rule slightly exceeds carbon content in natural shungite because it additionally contains finely-dispersed particles of oxides.

It means that unlike the prototype where production output makes maximum $0.45 \mathrm{~g} / 1000 \mathrm{~g}$ of natural shungite using the offered method concentrate recovery reaches significant values up to dozens of grams. Such high recovery is ensured by using sedimentological residual instead of non-filtration one as well as siphon separation of shungite suspension of residual. This totally excludes considerable absorption losses unavoidable when using filters. In practice the offered method is implemented as follows (Stechyshyn et al., 2008).

\section{MATERIALS AND METHODS}

Experiments: In sedimentation column (Fig. 1) which is tube 1 soldered on one side $50 \mathrm{~mm}$ in diameter and about $1 \mathrm{~m}$ long. The column is fixed in position with holder 2 to rack 5. Shungite at the rate of 200 grams is filled in the column preliminary crushed in a vibration disintegrator 75T-DRM to fineness not more than 1-5 $\mu$ and poured with $0.8 \mathrm{dm}, 3$ of distillate (concentration 20\%) where upon the column is tightly closed with plug 3 and the following experiments are carried out.

The column is turned through $180^{\circ}$ around axis 4 and held for $5 \mathrm{~min}$ during this time the largest and solid shungite particles sedimentologically gravitate to the column bottom (bottom sediment). In $5 \mathrm{~min}$ the column is turned through $180^{\circ}$ that is put in the initial position. Experiments 1 and 2 are repeated at least 3 times whereupon the suspension is allowed to settle during $2 \mathrm{~h}$

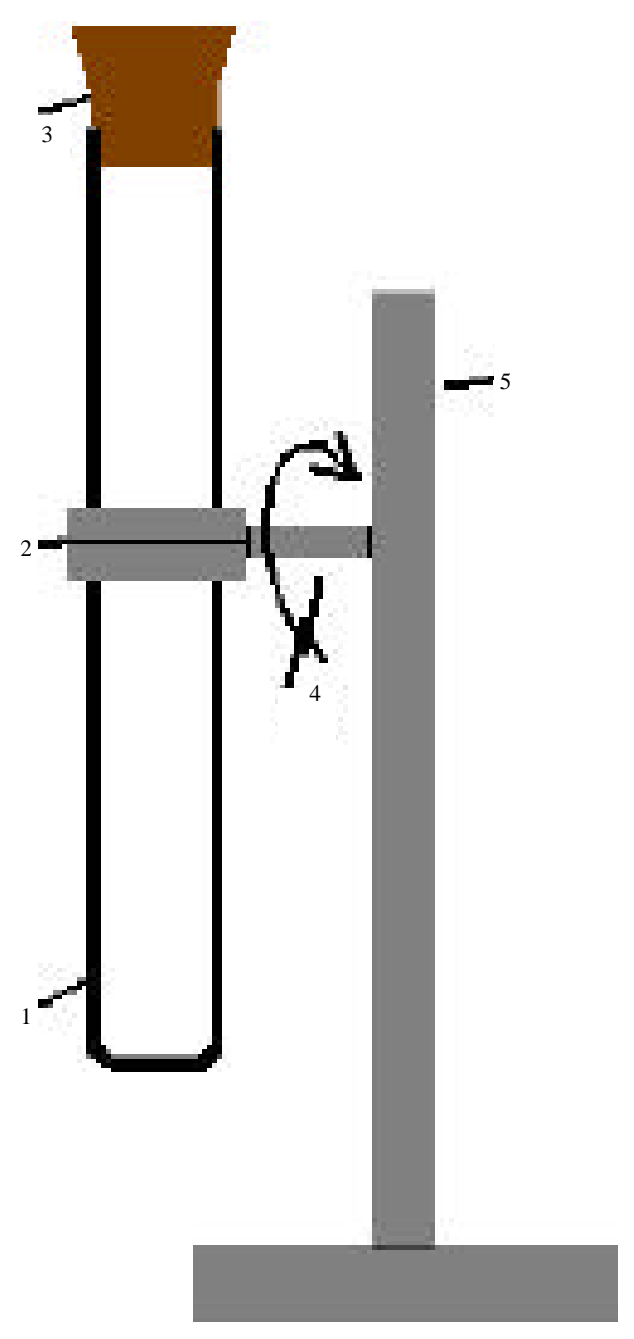

Fig. 1: Sedimentation column

whereas thickness of CEC concurrently recovered achieves 100-120 $\mu$. Upon expiration of $2 \mathrm{~h}$ the column plug shall be opened and the suspension is siphon separated of the residuals at the column bottom. Recovered suspension undergoes evaporation and annealing at temperature $473-523 \mathrm{~K}$ till a dry concentrate is obtained. Recovered concentrate is weighed with up to $10-3 \mathrm{~g}$ accuracy. The residuals remaining at the column bottom are poured with $0.8 \mathrm{dm} 3$ of distillate and the pervious experiment is repeated by points $1-6$.

Experiments by points 1-7 are repeated until the suspension becomes transparent and mass of recovered shungite concentrate is negligible. This is evidence of almost full recovery of carbonic component of natural shungite. Whole recovered suspension undergoes evaporation and the obtained concentrate undergoes annealing at temperature $473-523 \mathrm{~K}$. Here with shungite 
rock concentrate containing all forms of carbon as well as finely dispersed particles of various oxides included in composition of the original shungite are recovered.

\section{RESULTS AND DISCUSSION}

Table 1 contains results of using the offered method showing dependency of sedimentated shungite mass (in grams) from the number of experiments. Obviously about 15-20 experiments hall beaccomplished to recover almost all shungite concentrate. In essence, the concentrate recovery in this case is washing out the most dispersed component of crushed natural shungite.

From Table 1 as the number of experiments increases the amount of recovered sedimentation shungite naturally decreases and after 15 times washing out only insignificant value $(0.854 \mathrm{~g})$ left in comparison with the shungite original mass $(200 \mathrm{~g})$. The result of total washing out is $37.533 \mathrm{~g}$ which is $18.77 \%$ in comparison with the shungite original mass.

Microphotographs (Fig. 2a, b) convincingly prove that shungite concentrate composition depends on the number of experiment that is the number of stirring and settling. As the number of experiments increases the most finely dispersed particles of shungite rock are left in the sampled shungite suspension (Fig. 2b).

Figure 3 shows electron probe microphotograph of shungite suspension after settling during $24 \mathrm{~h}$. The suspension contains about $50 \%$ of particles not larger than $100 \mathrm{~nm}$ in size.

To recover nano-CEC natural shungite shall be duly prepared that is a concentrate shall be recovered. As it 's shown from Fig. 2, shungite concentrate recovered under different conditions has different dispersity and microstructure relatively coarse (Fig. 2a) and fine (Fig. 2b). Detailed researches show that the former contains the latter in its composition fully, since, the latter is separated from the former through additional only time consuming. As it is seen more finely, dispersed concentrate consists of light and dark particles predominantly spherical form smaller than $100 \mathrm{~nm}$ in size at the same time the light particles are considerably larger than the dark particles. Relatively large light particles in comparison with smaller and darker particles have increased tendency to create clusters. Results of studying the particle composition prove that light large particles predominantly consist of silicon dioxide whereas small particles-primarily of carbon. Studying fractional composition of shungite concentrate using Atomic Force Microscopy (AFM) method, showed that overwhelming majority of particles are smaller than $100 \mathrm{~nm}$ in size. It can be seen from histogram of particles
Table 1: Results of sedimentational selection of $200 \mathrm{~g}$ of shungite being crushed in a vibration disintegrator (sedimentation settling time is $2 \mathrm{~h}$ )

\begin{tabular}{lccc}
\hline & $\begin{array}{l}\text { Shungite original } \\
\text { mass for each } \\
\text { No. of } \\
\text { experiment }\end{array}$ & $\begin{array}{l}\text { Sedimentated } \\
\text { shungite mass } \\
\text { in each } \\
\text { experiment (g) }\end{array}$ & $\begin{array}{l}\text { Total } \\
\text { sedimentated } \\
\text { shungite } \\
\text { mass (g) }\end{array}$ \\
\hline 1 & 200.000 & 5.336 & 5.336 \\
2 & 194.663 & 3.965 & 9.301 \\
3 & 191.332 & 3.330 & 12.631 \\
4 & 187.366 & 3.348 & 15.982 \\
5 & 184.018 & 2.335 & 18.317 \\
6 & 181.698 & 2.335 & 20.653 \\
7 & 178.598 & 2.570 & 23.223 \\
8 & 176.984 & 2.700 & 25.923 \\
9 & 173.414 & 2.720 & 28.643 \\
10 & 171.079 & 2.614 & 31.257 \\
11 & 168.774 & 1.732 & 32.985 \\
12 & 167.042 & 1.498 & 34.483 \\
13 & 165.544 & 1.272 & 35.755 \\
14 & 184.272 & 0.921 & 36.676 \\
15 & 163.351 & 0.854 & 37.533 \\
16 & 162.497 & & \\
\hline
\end{tabular}

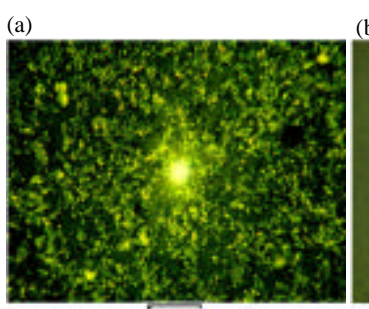

$10(\mathrm{mcm})$

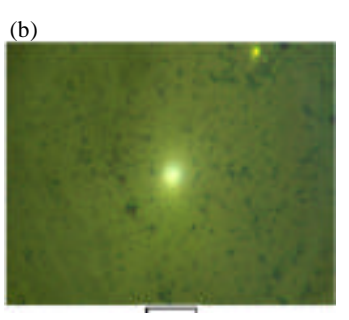

$10(\mathrm{mcm})$
Fig. 2: Optical photos of sedimentated particles: a) 2-time setting and b) 14-time setting (a)

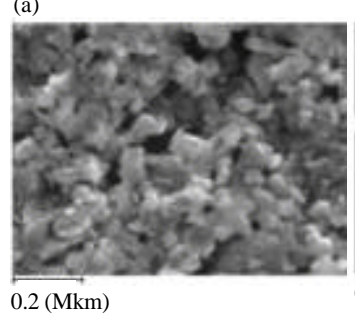

(b)

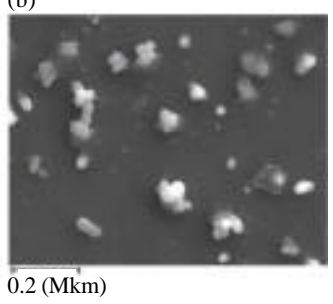

Fig. 3: Electron microscope photos of shungite concentrate recovered under different conditions

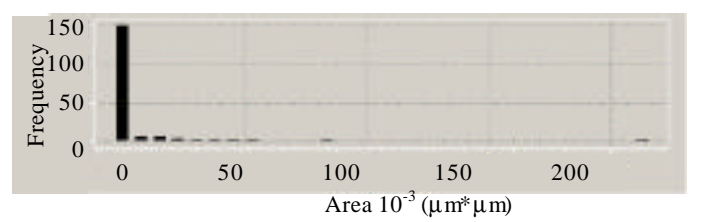

Fig. 4: AFM-histogram of particles used for recovering nano-CEC chromium shungite

in Fig. 4 and photograph of particles of shungite concentrate from the surface (Fig. 5a) and in 3D-imaging format (Fig. 5b). 



Fig. 5: AFM-photograph of particles of shungite concentrate recovered through sedimentation settling during 2 h: a) From the surface and b) In 3D-imaging format

\section{CONCLUSION}

As a result of accomplished studies the following conclusion was drawn: based on the review of contemporary state of knowledge about shungite a science-based idea of advisability to use shungite as a disperse phase in CEC composition for the first time was proposed. Three methods for preparing electrolyte to settle nano-CEC chromium shungite washing out method, dispersion method and method of recovering ready electrolyte suspension were developed. Application for an invention was submitted and concerning one of the variants accepted. In as much as natural shungite apart from various forms of carbonic component contains a large quantity of oxide impurities with dispersivity within the range from smaller than $100 \mathrm{~nm}$ to few microns large then chromium shungite coating may be named a nano-polycomposite coating.

\section{REFERENCES}

Anonymous, 2014. Composite electrolytic coatings. Patent No: 4-172-771, C25D 15-00, USA.

Cherdyntsev, V.V. and Y.M. Kolesnikov, 1965. [Ancient atmospheric argon in sedimentary rocks and minerals (In Russian)]. Dokl. AN. SSSR., 165: 1405-1408.

Cholodkevich, A.M., 1993. Reclamation of natural fullerenes from Karelia shungites. Rep. Acad. Sci., 330: $340-341$.
Sarsembinov, S.S., S.K. Yar-Mukhamedov and G.S. Yar-Mukhamedova, 2006. Physical Bases of Structure of Forming Composite Materials with Set Properties. Al-Farabi KazNU, Almaty, Kazakhstan, Pages: 404.

Sokolov, V.A. and Y.K. Kalinin, 1975. Shungites of Karelia and Possibilities of its Utilization. Karelia, Petrozavodsk, Russia, Pages: 240 (In Russian).

Stechyshyn, M.S., J.M. Bilyk and A.V. Martynyuk, 2008. Device for applying composite electrolytic coatings. Bull. KNU. Tech. Sci., 2: 196-199.

Tseluikin, V.N., 2005. Composite coatings with fullerene C60: Hydrogen materials science and chemistry of carbon nanomaterials. Proceedings of the 9th International Conference on Hydrogen Materials Science and Chemistry of Carbon Nanomaterials (ICHMS'05), September 5-11, 2005, Institute for Problems of Materials Science of N AS, Kiev, Ukraine, pp: 520-523.

Volkova, I.B. and M.V. Bogdanova, 1986. Petrology and genesis of Karelian shungite-high rank coal. Intl. J. Coal Geol., 6: 369-379.

Yar-Mukhamedov, S.H. and G.S. Yar-Mukhamedova, 2010. Transfomation of Composite Electrolytic Coatings Formation Conditions. Tsvetnye Metally, Moscow, Russia,.

Yar-Mukhamedov, S.K. and G.S. Yar-Mukhamedova, 2001. Contemporary state and development perspectives for composite coatings in machine building. Vestnik KazNTU, 4: 82-89. 